\title{
Magnetohydrodynamic Fluid Flow due to an Unsteady Stretching Sheet with Thermal Radiation, Porous Medium, and Variable Heat Flux
}

\author{
Ahmed M. Megahed $\left(D,{ }^{1}\right.$ Nourhan I. Ghoneim, ${ }^{2}$ M. Gnaneswara Reddy, ${ }^{3}$ \\ and Mostafa El-Khatib ${ }^{4}$ \\ ${ }^{1}$ Department of Mathematics, Faculty of Science, Benha University, Benha, Egypt \\ ${ }^{2}$ Nautical Department-Marine Engineering, International Maritime College Oman (IMCO), Suhar, Oman \\ ${ }^{3}$ Department of Mathematics, Acharya Nagarjuna University Campus, Ongole 523001, India \\ ${ }^{4}$ Basic Sciences Department, Faculty of Engineering, Pharos University in Alexandria, Alexandria, Egypt
}

Correspondence should be addressed to Ahmed M. Megahed; ahmed.abdelbaqk@fsc.bu.edu.eg

Received 17 October 2020; Revised 23 December 2020; Accepted 7 January 2021; Published 31 January 2021

Academic Editor: Jose Gaite

Copyright (C) 2021 Ahmed M. Megahed et al. This is an open access article distributed under the Creative Commons Attribution License, which permits unrestricted use, distribution, and reproduction in any medium, provided the original work is properly cited.

\begin{abstract}
A shooting method has been introduced for determining the numerical solution of the ordinary differential equations which describe the Newtonian magnetohydrodynamic laminar fluid flow due to an unsteady stretching sheet together with the presence of thermal radiation and variable heat flux. The variable viscosity and variable conductivity are taken into consideration. Absence of magnetic field in some studies restricts the development of the energy-efficient heat transfer mechanism as is desired in numerous applications. The present study encompasses parameters such as unsteadiness parameter, porous parameter, viscosity parameter, magnetic number, radiation parameter, and conductivity parameter. It has been consummated that the proposed model is superior to other existing models for the industrial fluid.
\end{abstract}

\section{Introduction}

Fluid flow and heat transfer mechanisms with the magnetohydrodynamic considerations play an important role in many astrophysical processes, space physics, and engineering $[1,2]$. As a result, there has been considerable interest in bringing accurate and reliable numerical methods concerning such heat and mass transfer phenomena. In this regard, several numerical methods were developed during the last five decades to solve these types of flow problems [3-5]. In industrial and engineering applications and in many branches of manufacturing operations and technology such as paper production, polymer processes, reactor fluidization, annealing and tinning of copper wires, and metal spinning [6-9], improvement of the thermal performance of the mechanism of heat transfer devices is often desired. One way of enhancing the performance of heat mechanism is to investigate some of the underlying important physical processes, which actually affect the heat transfer surface $[10,11]$. A generalization for the fluid flow models regarding the unsteady flow can be found in [12-17]. Another important phenomenon that can actually affect the heat process mechanism is the heat flux. A number of studies [18-20] have therefore been introduced on these topics and it has been shown that the fluid flow together with the heat flux is very important in some of the manufacturing processes. Liu et al. [21] were the pioneers in innovating the variable heat flux model which coincides with the unsteady flow models under various conditions. However, our knowledge of the precise nature of heat transfer mechanism is still incomplete.

One of the objectives of the current study is to introduce an elaboration, as complete as possible, of the variable fluid properties on the laminar MHD Newtonian fluid flow and heat transfer past an unsteady stretching sheet which is 
embedded in a porous medium, and especially in the presence of variable heat flux.

\section{Mathematical Formulation}

The basic idea of this section is to introduce an adequate description, as complete as possible, for all equations that administer the flow of laminar boundary layer fluid for an incompressible two-dimensional viscous flow and heat transfer due to an unsteady stretching sheet.

The fine study is easier to describe even with the physical properties that can be taken into account, such as the surface velocity $U_{w}(x, t)$, the fluid viscosity $\mu$, and the fluid thermal conductivity $\kappa$, since they are assumed to be universal. This model involves the variable heat flux $q(x, t)$, which is previously introduced in detail by Liu et al. [21], and is given as follows:

$$
q(x, t)=-\kappa \frac{\partial T}{\partial y}=T_{0} \frac{\mathrm{d} x^{r}}{(1-a t)^{m+(1 / 2)}},
$$

where $T_{0}$ is a reference temperature, $a$ is a positive constant, $d$ is a constant, and $r$ and $m$ are space and time indices, respectively. Further, the applied transverse magnetic field can be introduced as follows [22]:

$$
B=B_{0}(1-a t)^{(-1 / 2)} \text {, }
$$

where $B_{0}$ is a constant. This special form for the applied transverse magnetic field will allow the existence of the dimensionless magnetic field parameter governing the flow velocity inside the boundary layer. Herein, we must mention that the MHD phenomenon is crucial owing to the wide applicability of the present consideration to other fields such as the medical science. It is a well-known fact that the human blood flow rate can be reduced by exerting an external magnetic field. So, the magnetic field can be used to regulate blood flow, in particular, during surgery [22]. Furthermore, we must refer that the choice for this form has also been studied by Mahmoud and Megahed [23] and Prasad et al. [24].

According to Liu et al. [21], the governing equations that describe the physical situation are expressed as follows:

$$
\begin{gathered}
\frac{\partial u}{\partial x}+\frac{\partial v}{\partial y}=0 \\
\frac{\partial u}{\partial t}+u \frac{\partial u}{\partial x}+v \frac{\partial u}{\partial y}=\frac{1}{\rho_{\infty}} \frac{\partial}{\partial y}\left(\mu \frac{\partial u}{\partial y}\right)-\frac{\sigma B^{2}}{\rho_{\infty}} u-\frac{\mu}{\rho_{\infty} k} u \\
\frac{\partial T}{\partial t}+u \frac{\partial T}{\partial x}+v \frac{\partial T}{\partial y}=\frac{1}{\rho_{\infty} c_{p}} \frac{\partial}{\partial y}\left(\kappa \frac{\partial T}{\partial y}\right)-\frac{1}{\rho_{\infty} c_{p}} \frac{\partial q_{r}}{\partial y},
\end{gathered}
$$

where $u$ and $v$ are the velocity components along the $x$ and $y$ directions, respectively. $t$ is the time, $\rho_{\infty}$ is the fluid density away from the sheet, $\sigma$ is the electrical conductivity, $k$ is the permeability of the porous medium, $T$ is the temperature of the fluid, $c_{p}$ is the specific heat at constant pressure, and $q_{r}$ indicates the radiative heat flux which is expressed in terms of $T$ as introduced previously by Prasad et al. [24] as follows:

$$
q_{r}=-\frac{4 \sigma^{*}}{3 k^{*}} \frac{\partial T^{4}}{\partial y},
$$

where $\sigma^{*}$ is the Stefan-Boltzmann constant and $k^{*}$ is the Rosseland mean absorption coefficient. It should be noted that, by using the Rosseland approximation, the present analysis is limited to optically thick fluids. If the temperature differences within the flow are sufficiently small, then equation (5) can be linearized by expanding $T^{4}$ into the Taylor series about $T$, and neglecting higher-order terms, we get

$$
T^{4} \approx 4 T_{\infty}^{3} T-3 T_{\infty}^{4} .
$$

Equations (3)-(5) are subject to the following boundary conditions:

$$
\begin{gathered}
u=U_{w}, \\
v=0, \\
-\kappa \frac{\partial T}{\partial y}=q(x, t) \quad \text { at } y=0, \\
u \longrightarrow 0, T \longrightarrow T_{\infty} \quad \text { as } y \longrightarrow \infty,
\end{gathered}
$$

where $T_{\infty}$ is the fluid temperature at the ambient and $U_{w}$ is the surface velocity which can be defined as

$$
U_{w}=\frac{b x}{1-a t}
$$

To preserve the dimension for the velocity $U$, we observe that both the positive constants $a$ and $b$ have dimension $t^{-1}$.

The governing equations (3)-(5) can be rewritten in the form by using the following variables:

$$
\begin{gathered}
\eta=\left(\frac{b}{v_{\infty}}\right)^{1 / 2}(1-a t)^{(-1 / 2)} y, \\
\psi=\left(\nu_{\infty} b\right)^{1 / 2}(1-a t)^{(-1 / 2)} x f(\eta), \\
\theta(\eta)=\frac{T-T_{\infty}}{\left(q(x, t) / \kappa_{\infty}\right) \sqrt{\nu_{\infty} / b}(1-a t)^{(1 / 2)}}
\end{gathered}
$$

where $\psi(x, y)$ is a stream function in which $u=\partial \psi / \partial y$ and $v=-(\partial \psi / \partial x)$. Also, $v_{\infty}$ is the kinematic viscosity at the ambient, $\theta(\eta)$ is the dimensionless temperature, and $f(\eta)$ is the dimensionless stream function.

In addition, the fluid viscosity $\mu$ and the fluid thermal conductivity $\kappa$ are considered to vary as a function of temperature as follows $[23,25]$ :

$$
\begin{aligned}
\frac{\mu}{\mu_{\infty}} & =e^{-\alpha \theta}, \\
\frac{\kappa}{\kappa_{\infty}} & =(1+\epsilon \theta),
\end{aligned}
$$

where $\mu_{\infty}$ is the viscosity at the ambient, $\alpha$ is the dimensionless viscosity parameter with $\alpha>0$ for liquid and $\alpha<0$ 
for gases, $\kappa_{\infty}$ is the thermal conductivity away from the surface, and $\epsilon$ is the thermal conductivity parameter.

Using equations (10) and (11), the continuity equation (3) is satisfied automatically, but equations (4) and (5) are then reduced to the system of highly nonlinear coupled ordinary differential equations given as follows:

$$
\begin{aligned}
e^{-\alpha \theta}\left(f^{\prime \prime \prime}-\alpha \theta^{\prime} f^{\prime \prime}\right)+f f^{\prime \prime}-f^{\prime 2}-S\left(\frac{\eta}{2} f^{\prime \prime}+f^{\prime}\right)-M f^{\prime}-\gamma e^{-\alpha \theta} f^{\prime} & =0, \\
\frac{1}{\operatorname{Pr}}\left[(1+R+\varepsilon \theta) \theta^{\prime \prime}+\varepsilon \theta^{\prime 2}\right]+f \theta^{\prime}-r f^{\prime} \theta-S\left(\frac{\eta}{2} \theta^{\prime}+m \theta\right) & =0 .
\end{aligned}
$$

Also, the transformed boundary conditions become

$$
\begin{aligned}
& f(0)=0, \\
& f^{\prime}(0)=1, \\
& \theta^{\prime}(0)=\frac{-1}{1+R+\epsilon \theta(0)}, \\
& \quad f^{\prime} \longrightarrow 0, \\
& \theta \longrightarrow 0 \text { as } \eta \longrightarrow \infty .
\end{aligned}
$$

The last part of equation (15) is due to the heat flux that basically depends on $R$ and $\varepsilon$ in which they serves as the radiation parameter and the conductivity parameter, respectively. Here, $M=\sigma B_{0}^{2} / b \rho_{\infty}$ is the magnetic number, $S=$ $a / b$ is the unsteadiness parameter, $\gamma=\mu_{\infty}(1-a t) / \rho_{\infty} k b$ is the local Darcy number, $R=16 \sigma^{*} T_{\infty}^{3} / 3 \kappa_{\infty} k^{*}$ is the radiation parameter, and $\operatorname{Pr}=\mu_{\infty} c_{p} / \kappa_{\infty}$ is the Prandtl number. Herein, it is worth mentioning that both the velocity field and temperature field are coupled to each other as we can observe from equations (13) and (14). On the other hand, it is noted that, for steady problem $(S=0)$, in the absence of thermal radiation $(R=0)$, magnetic field, and porous parameter $(M=\gamma=0)$ and the viscosity not a function of temperature $(\alpha=0)$, the velocity field was solved analytically by Crane [26] with $f=1-e^{-\eta}$.

The most important characteristics of fluid flow which can be appeared in the part of resistance force or the frictional drag between the stretching sheet and the moving fluid are the nondimensional local skin-friction $\left(C f_{x}\right)$ or the frictional drag coefficient which is defined by [21]

$$
C f_{x}=\frac{2 \tau_{w}}{\rho U_{w}^{2}},
$$

where $\tau_{w}=-[\mu(\partial u / \partial y)]_{y=0}$.

Additionally, cooling process is one of the most important engineering applications ranging from the cooling of the food products to hot processing of solid metals. Mathematically, the factor which can measure this important process is the local Nusselt number $\left(N u_{x}\right)$ defined as follows [21]:

$$
N u_{x}=\frac{x q(x, t)}{\kappa_{\infty}\left(T_{w}-T_{\infty}\right)},
$$

where $T_{w}=T_{\infty}+T_{0}\left(\mathrm{~d} x^{r} / \kappa_{\infty} \sqrt{\left(b / \nu_{\infty}\right)}\right)(1-a t)^{-m} \theta(0)$.

Using the nondimensional equations (10) and (11), the local skin-friction coefficient and local Nusselt number can be written as

$$
\begin{aligned}
C f_{x} \frac{\operatorname{Re}_{x}^{(1 / 2)}}{2} & =-e^{-\alpha \theta(0)} f^{\prime \prime}(0), \\
N u_{x} \operatorname{Re}_{x}^{(-1 / 2)} & =\frac{1}{\theta(0)},
\end{aligned}
$$

where $\operatorname{Re}_{x}=U_{w} x / \nu_{\infty}$ is the local Reynolds number. It is obvious from the above equation (17) that the local Nusselt number $N u_{x}$ is reciprocal to $\theta(0)$. On the other hand, from the same equation, it is clear that the local skin-friction coefficient is proportional to $f^{\prime \prime}(0)$ and it also depends on the surface temperature $\theta(0)$ and the viscosity parameter $\alpha$.

\section{Numerical Solution}

The common techniques for solving boundary value problems are linear and nonlinear shooting methods [27-29]. Therefore, in this section, we are primarily interested in shooting techniques. Shooting method has been employed to solve the Newtonian fluid flow model defined by equations (13) and (14) with boundary conditions (15) and (16). For the numerical integration, we use the fourthorder Runge-Kutta method. To proceed, we define the following:

$$
\begin{aligned}
& y_{1}=f, \\
& y_{2}=y_{1}^{\prime}, \\
& y_{3}=y_{2}^{\prime}, \\
& y_{4}=\theta, \\
& y_{5}=y_{4}^{\prime} .
\end{aligned}
$$

Equations (13) and (14) are then reduced to a system of first-order ordinary differential equations, i.e., 


$$
\begin{aligned}
y_{1}^{\prime} & =y_{2}, \\
y_{1}(0) & =0 \\
y_{2}^{\prime} & =y_{3} \\
y_{2}(0) & =1 \\
y_{3}^{\prime} & =\alpha y_{5} y_{3}+e^{\alpha y_{4}}\left(y_{2}^{2}-y_{1} y_{3}+S\left(\frac{\eta}{2} y_{3}+y_{2}\right)+M y_{2}\right)+\gamma y_{2}, \quad y_{3}(0)=\varepsilon_{1}, \\
y_{4}^{\prime} & =y_{5}, \quad-1 \\
y_{5}(0) & =\frac{1+R+\epsilon y_{4}(0)}{1+}, \\
y_{5}^{\prime} & =\frac{1}{1+R+\epsilon y_{4}}\left(\operatorname{Pr}\left(r y_{2} y_{4}-y_{1} y_{5}+S\left(\frac{\eta}{2} y_{5}+m y_{4}\right)\right)-\epsilon y_{5}^{2}\right), \quad y_{5}(0)=\varepsilon_{2},
\end{aligned}
$$

where $\varepsilon_{1}$ and $\varepsilon_{2}$ are determined such that the outer boundary conditions $y_{2}(\infty)$ and $y_{4}(\infty)$ are satisfied. The shooting method is used to guess $\varepsilon_{1}$ and $\varepsilon_{2}$ by iteration until the outer boundary conditions are satisfied. The resulting differential equations can be integrated by fourth-order Runge-Kutta integration scheme. The above procedure is repeated until we get the results up to the desired degree of accuracy, $10^{-5}$.

\section{Validation of the Proposed Method}

To assess the validity and accuracy of the present numerical scheme, some wall temperature values $\theta(0)$ for some values of thermal conductivity parameter and Prandtl number have been compared to those of the earlier steady-state problems of Prasad et al. [30] and the results of comparison are given in Table 1. Clearly our results are in excellent agreement with those of Prasad et al. [30].

\section{Results and Discussion}

There is potentially a wide parameter domain to scout, but we observe that the computations for larger values of governing parameters are particularly complicated because of inability of numerical convergence. Consequently, in this section, we present results for a very finite domain of physical parameters, chosen to explain the main trends. Influence of magnetic parameter, unsteadiness parameter, radiation parameter, viscosity parameter, Darcy number, Prandtl number, and variable thermal conductivity is discussed in this section. The flow parameter values are $\alpha=$ $\epsilon=r=m=0.4, R=1.0, S=0.5, M=0.5, \operatorname{Pr}=0.71$ as input to acquire the results for complete production. The results are enumerated with Figures 1-10. Figures 2(a) and 2(b) show the deviations of magnetic parameter on velocity and temperature profiles. From these figures, it is clear that the temperature profile rises for boost up values of $M$. Figure 2(a) shows that velocity decelerates with inflation in magnetic parameter. In general, the increment in Hartmann number generates the Lorentz force known as resistive force.
The influence of different values of $R$ on thermal and velocity profiles is shown in Figures 3(a) and 3(b). It shows that the thermal boundary layer thickness and temperature distribution are improved for increasing values of $R$. Higher values of $R$ afford more temperature to the fluid flow which generates an increment in temperature and thermal boundary layer thickness and the opposite effect is seen in Figure 3(b).

The velocity and temperature profiles for various values of the parameter $\gamma$ are shown in Figures 4(a) and 4(b), respectively. A large value of $\gamma$ parameter corresponds to a low velocity distribution and high temperature distribution as illustrated from both figures.

The numerical results given in Figures 5(a) and 5(b) show that the velocity distribution diminishes with enhancement of $\alpha$, while the sheet temperature $\theta(0)$ and the dimensionless temperature increases with increase in the viscosity parameter $\alpha$.

Figures $6(\mathrm{a})$ and $6(\mathrm{~b})$ depict the velocity profile $f^{\prime}(\eta)$ and the temperature profile $\theta(\eta)$ for varying the unsteadiness parameter $S$. It is interesting to note that the temperature presents a maximum distribution at small unsteadiness parameter $S$ via the imposed thermal boundary condition. The velocity profiles show a similar trend.

The influence of different values of thermal conductivity parameter $\epsilon$ on both velocity and temperature profiles is shown in Figures $7(\mathrm{a})$ and $7(\mathrm{~b})$. It is revealed from Figure $7(\mathrm{a})$ that the velocity $f^{\prime}(\eta)$ enhanced when we escalate the values of $\epsilon$. Also, from Figure 7(b), it can be deduced that a high conductivity parameter $\epsilon$ causes a considerable increase of the temperature beside the sheet.

To get a clear view of the flow field, the stream line patterns are plotted in Figures 8-10 with different values of magnetic parameter $M$, unsteady parameter $S$, and Darcy number. Figures $8(\mathrm{a})$ and $8(\mathrm{~b})$ show the effect of $M$ on stream line pattern. Overstock of stream lines is observed as $M$ diminished. Figures 9(a) and 9(b) exhibit the impact of $S$ on stream line pattern. Clearly, a retardation in the flow pattern is observed as $S$ enhanced. Figures 10(a) and 10(b) 
TABLE 1: Comparison of wall temperature $\theta(0)$ values when $\alpha=M=\gamma=S=0, r=1$ with Prasad et al. [30] (Newtonian case ( $n=1), \beta=0$, and $\epsilon=0.0,0.1)$.

\begin{tabular}{lccc}
\hline$\epsilon$ & Pr & Prasad et al. [30] & Present work \\
\hline 0.0 & 0.7 & 1.2470 & 1.2490 \\
0.0 & 1.0 & 0.9986 & 0.9991 \\
0.0 & 2.0 & 0.6575 & 0.6576 \\
0.0 & 5.0 & 0.3922 & 0.3922 \\
0.1 & 0.7 & 1.3714 & 1.3712 \\
0.1 & 1.0 & 1.0758 & 1.0757 \\
0.1 & 2.0 & 0.6894 & 0.6891 \\
0.1 & 5.0 & 0.4032 & 0.4032 \\
\hline
\end{tabular}

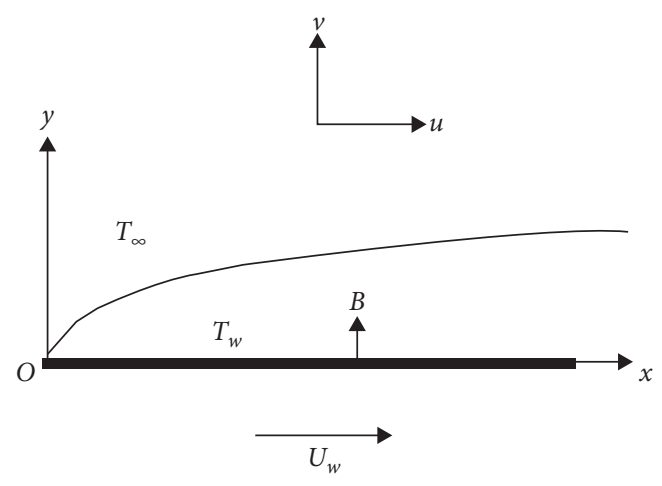

FIgure 1: Physical model and coordinate system.

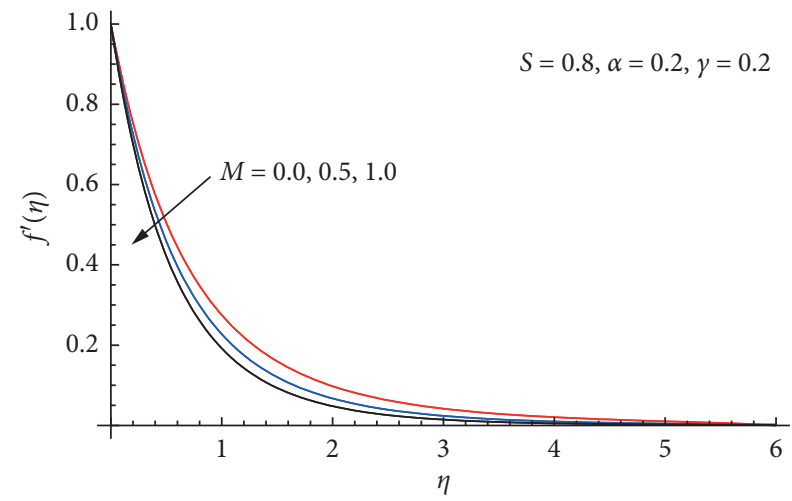

(a)

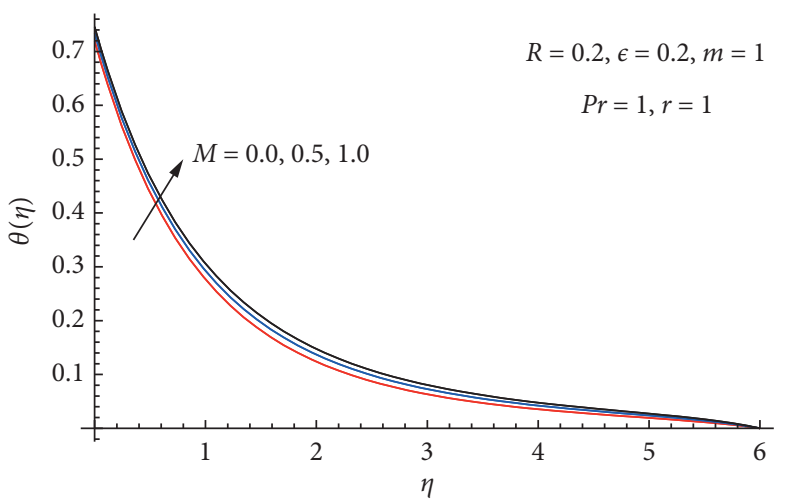

(b)

FIgURE 2: (a) Velocity distribution for $M$. (b) Temperature distribution for $M$.

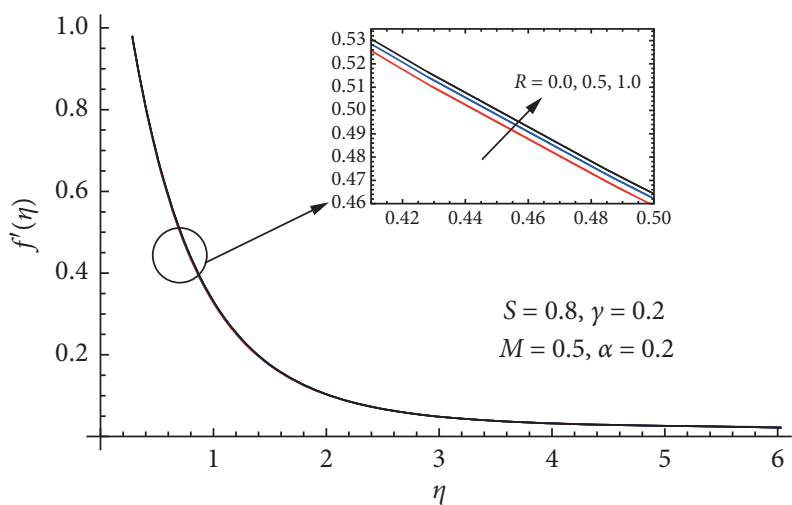

(a)

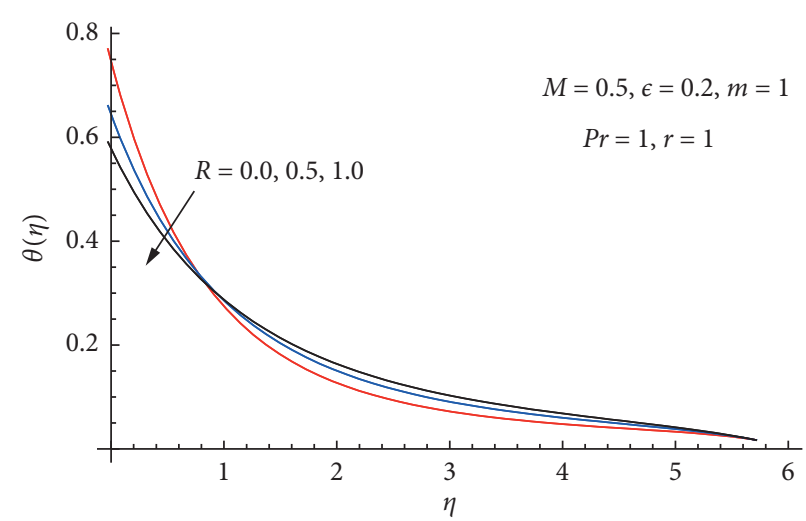

(b)

Figure 3: (a) Velocity distribution for $R$. (b) Temperature distribution for $R$. 


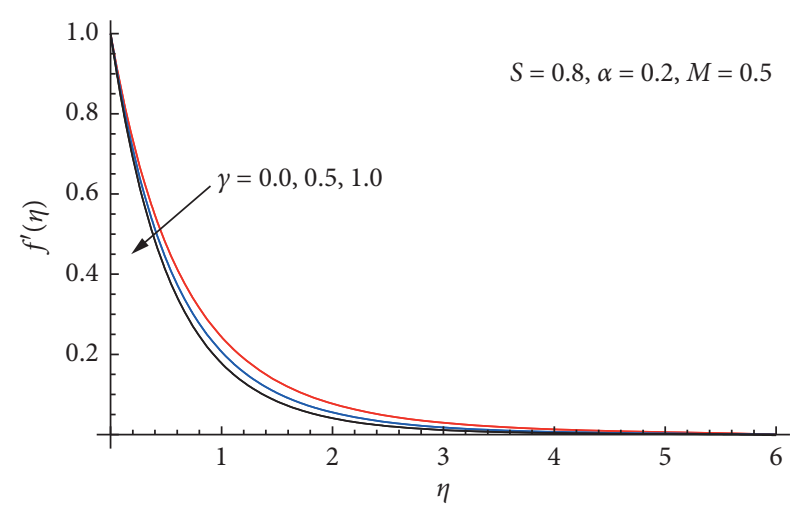

(a)

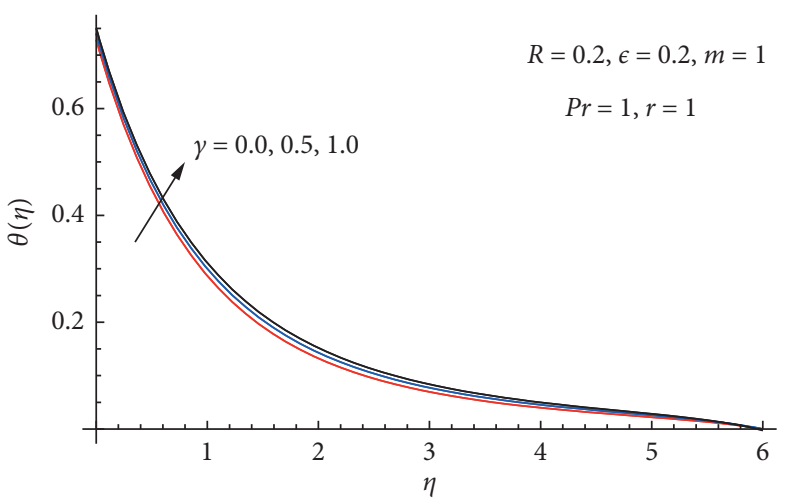

(b)

FIgURE 4: (a) Velocity distribution for $\gamma$. (b) Temperature distribution for $\gamma$.

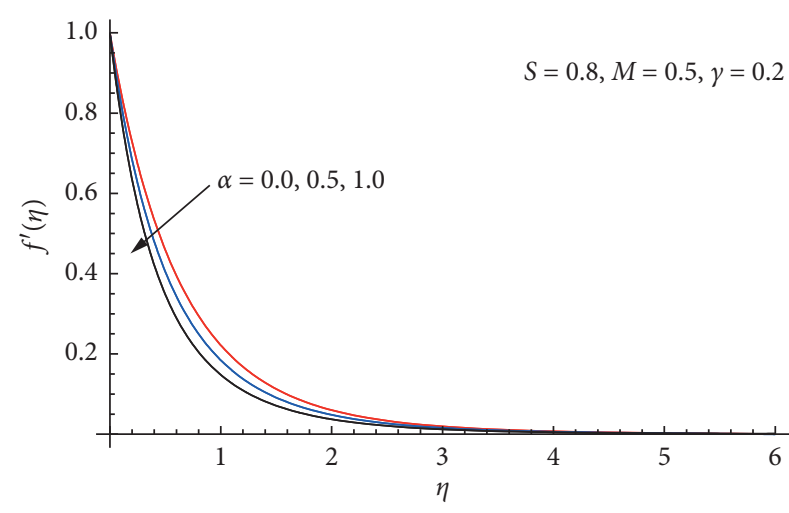

(a)

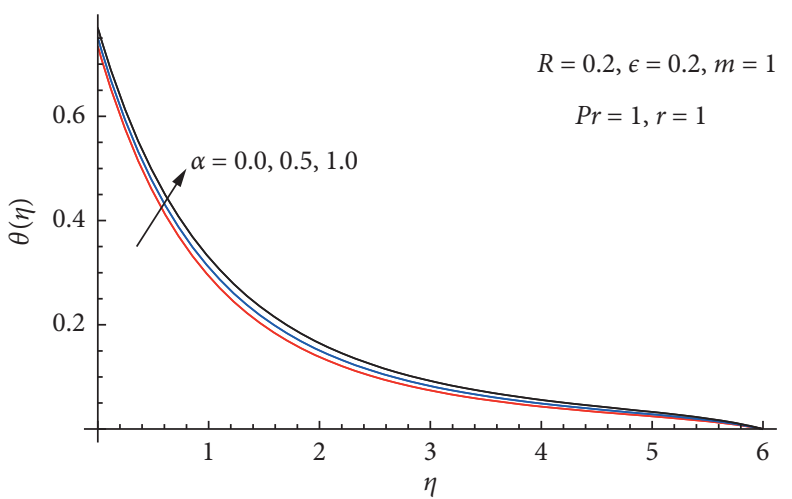

(b)

Figure 5: (a) Velocity distribution for $\alpha$. (b) Temperature distribution for $\alpha$.

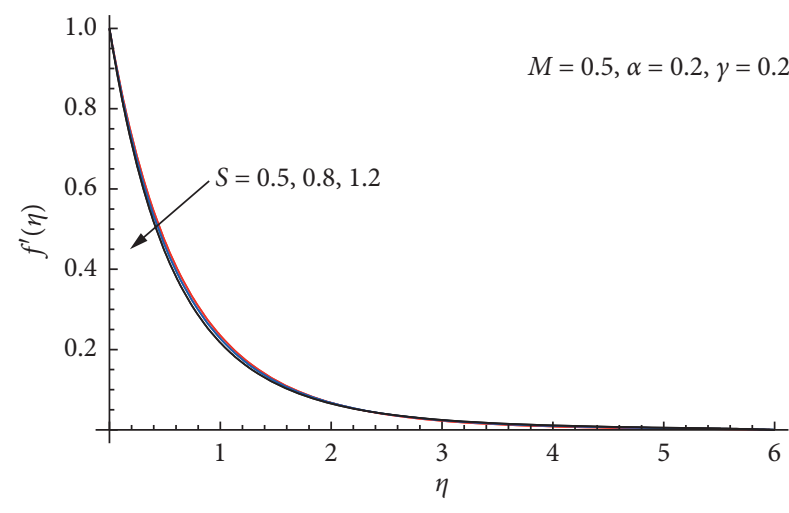

(a)

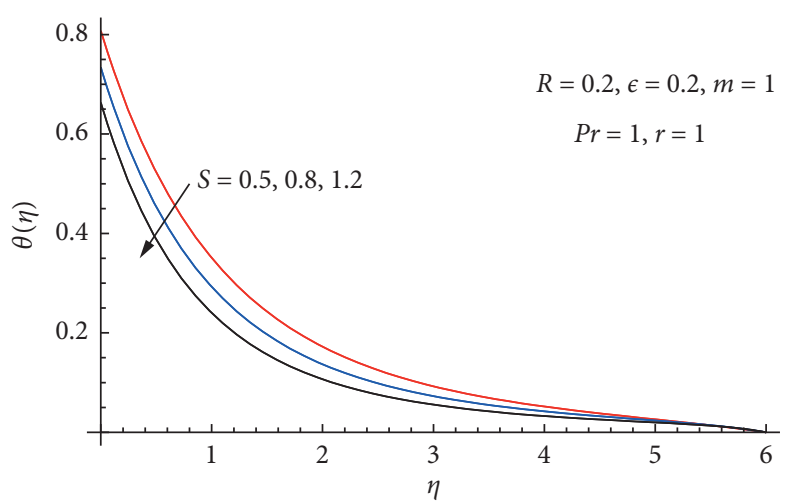

(b)

FIgure 6: (a) Velocity distribution for $S$. (b) Temperature distribution for $S$.

show the effect of $\gamma$ on the stream line pattern. The same behavior of the stream line pattern for altering the parameter is similar to the magnetic parameter $M$.

Table 2 is presented to elucidate the behavior of the local skin-friction coefficient $C f_{x}\left(\operatorname{Re}_{x}^{(1 / 2)} / 2\right)$ and the local Nusselt number $N u_{x} \operatorname{Re}_{x}^{(-1 / 2)}$ with changes in thermal conductivity parameter $\epsilon$, viscosity parameter $\alpha$, unsteadiness parameter $S$, radiation parameter $R$, and the magnetic number $M$. We can then see from Table 2 that the local skin-friction coefficient increases with the increase of unsteadiness 


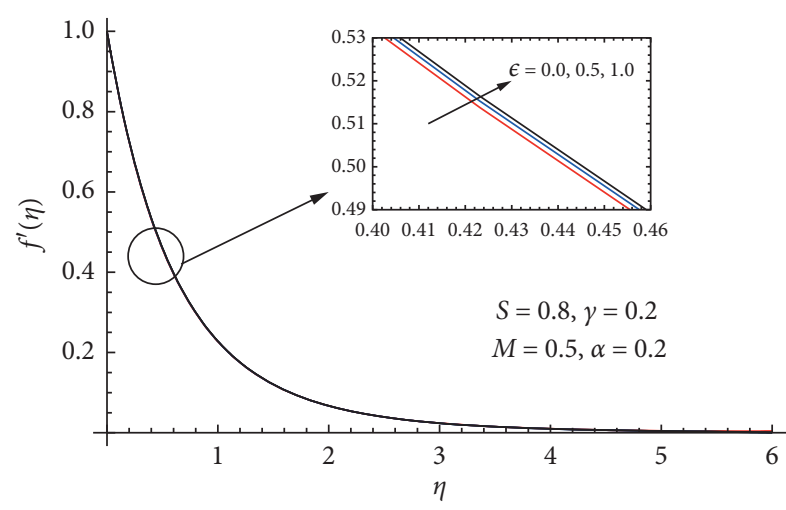

(a)

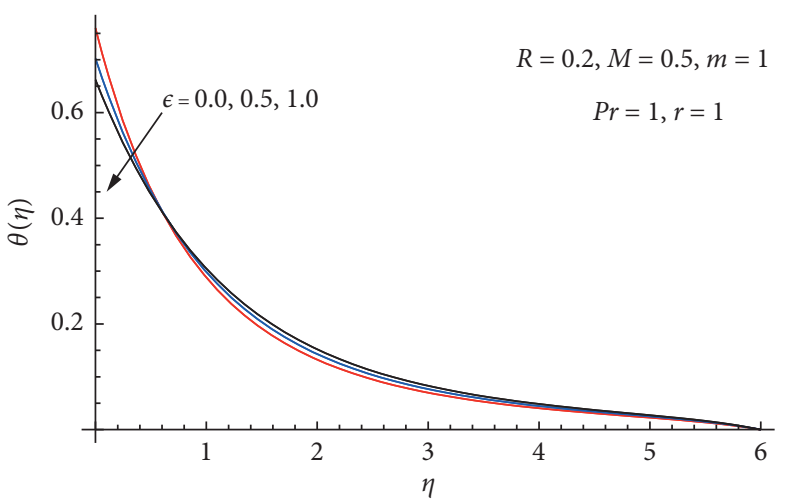

(b)

Figure 7: (a) Velocity distribution for $\epsilon$. (b) Temperature distribution for $\epsilon$.

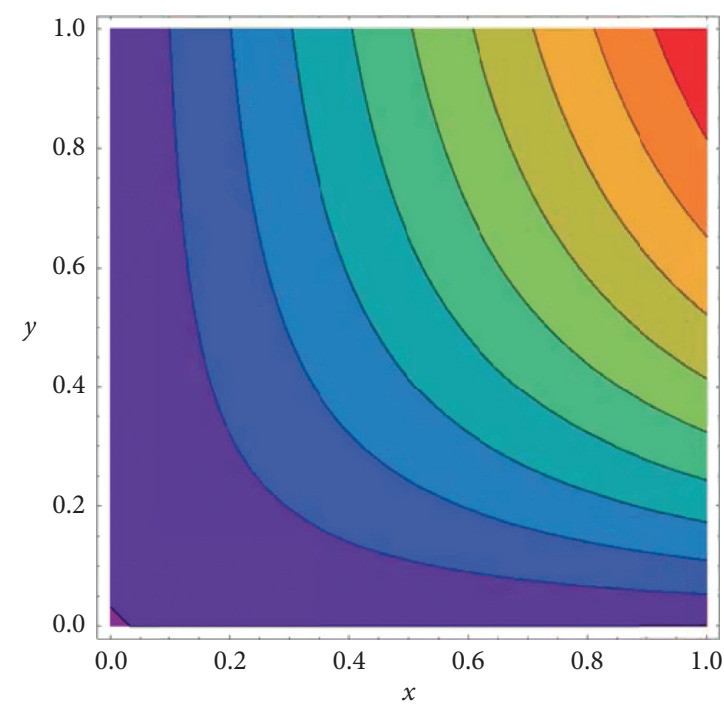

(a)

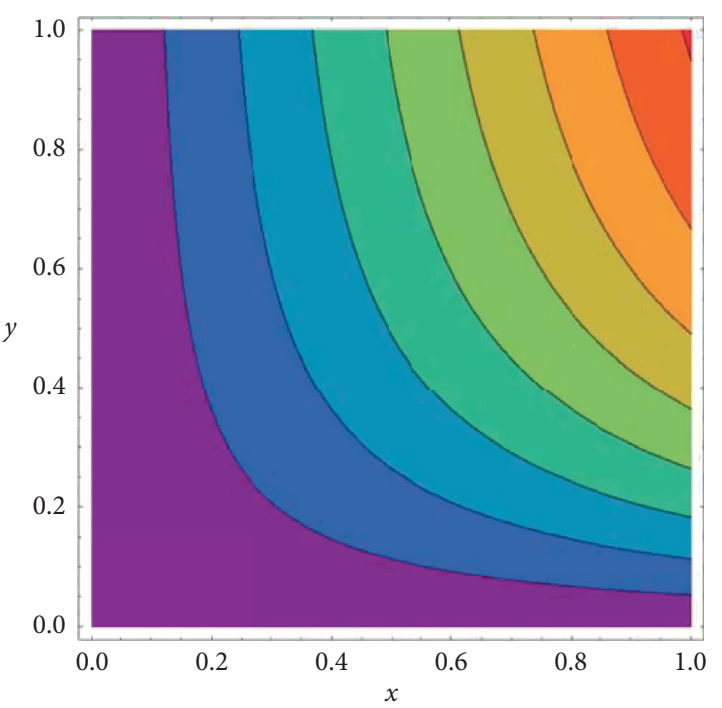

(b)

Figure 8: (a) Stream lines for $M=0.5$. (b) Stream lines for $M=1.0$.

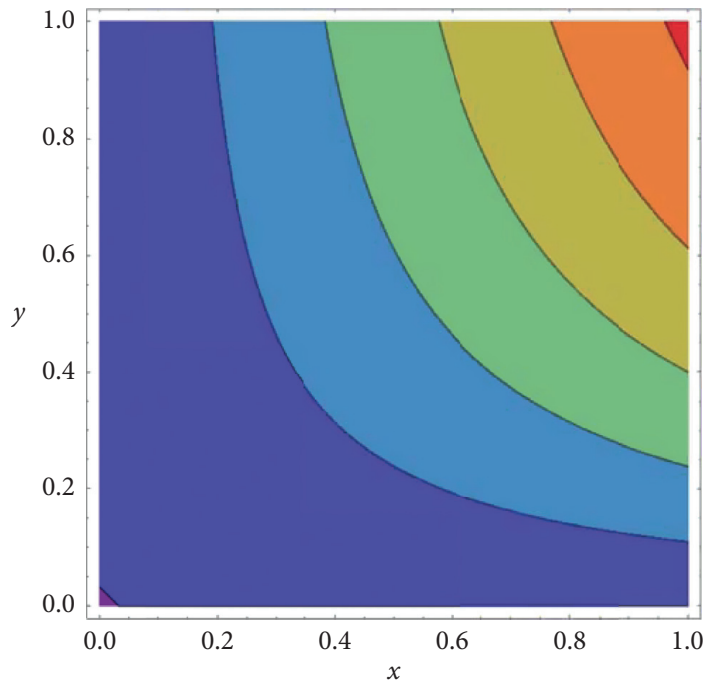

(a)

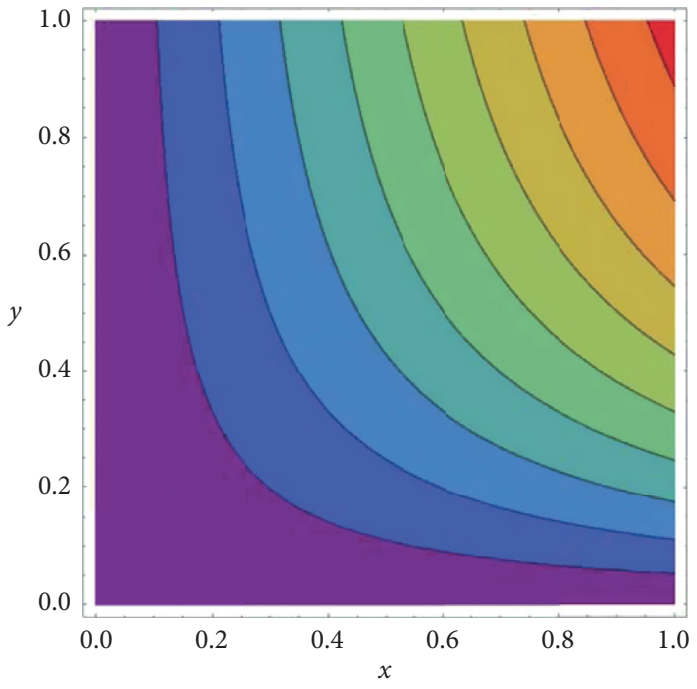

(b)

FIgURE 9: Stream lines for $S=0.0$. (b) Stream lines for $S=1.0$. 


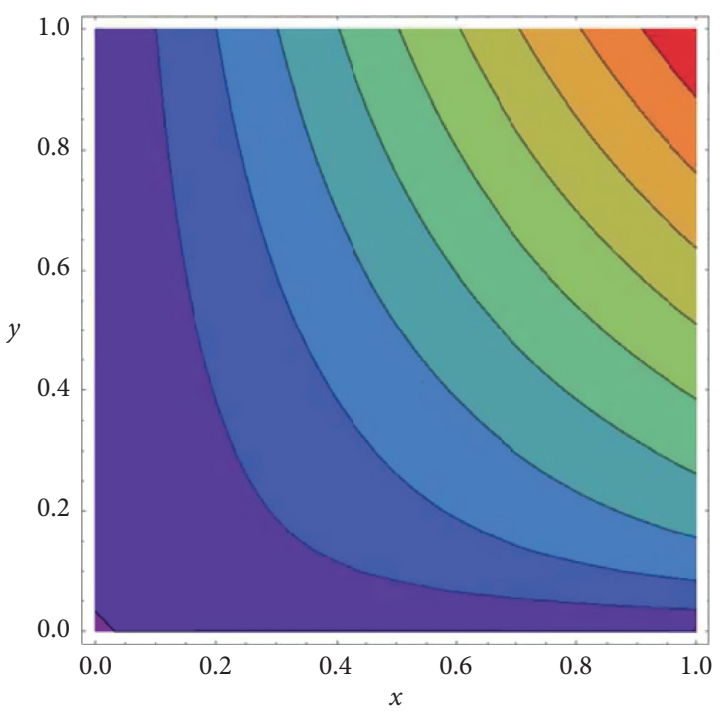

(a)

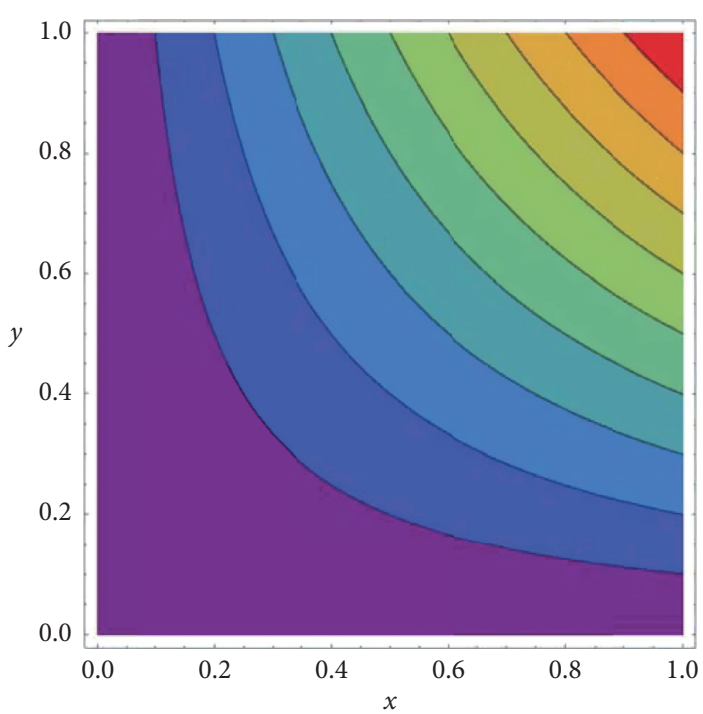

(b)

Figure 10: (a) Stream lines for $\gamma=0.5$. (b) Stream lines for $\gamma=2.0$.

TABLE 2: Variation of $-C f_{x}\left(\operatorname{Re}_{x}^{(1 / 2)} / 2\right)$ and $N u_{x} \operatorname{Re}_{x}^{(-1 / 2)}$ for various values of $S, \alpha, M, \epsilon, \gamma$, and $R$ with $r=m=\operatorname{Pr}=1$.

\begin{tabular}{cccccccc}
\hline$M$ & $R$ & $\gamma$ & $\alpha$ & $S$ & $\epsilon$ & $-C f_{x}\left(\mathrm{Re}_{x}^{(1 / 2)} / 2\right)$ & $N u_{x} \mathrm{Re}_{x}^{(-1 / 2)}$ \\
\hline 0.0 & 0.2 & 0.2 & 0.2 & 0.8 & 0.2 & 1.25980 & 1.38944 \\
0.5 & 0.2 & 0.2 & 0.2 & 0.8 & 0.2 & 1.42306 & 1.36269 \\
1.0 & 0.2 & 0.2 & 0.2 & 0.8 & 0.2 & 1.56849 & 1.34093 \\
0.5 & 0.0 & 0.2 & 0.2 & 0.8 & 0.2 & 1.41848 & 1.27138 \\
0.5 & 0.5 & 0.2 & 0.2 & 0.8 & 0.2 & 1.42861 & 1.48658 \\
0.5 & 1.0 & 0.2 & 0.2 & 0.8 & 0.2 & 1.43564 & 1.66778 \\
0.5 & 0.2 & 0.0 & 0.2 & 0.8 & 0.2 & 1.36686 & 1.37188 \\
0.5 & 0.2 & 0.5 & 0.2 & 0.8 & 0.2 & 1.50277 & 1.35021 \\
0.5 & 0.2 & 1.0 & 0.2 & 0.8 & 0.2 & 1.62566 & 1.33214 \\
0.5 & 0.2 & 0.2 & 0.0 & 0.8 & 0.2 & 1.51445 & 1.37507 \\
0.5 & 0.2 & 0.2 & 0.5 & 0.8 & 0.2 & 1.29007 & 1.34321 \\
0.5 & 0.2 & 0.2 & 1.0 & 0.8 & 0.2 & 1.08033 & 1.30834 \\
0.5 & 0.2 & 0.2 & 0.2 & 0.5 & 0.2 & 1.34063 & 1.23844 \\
0.5 & 0.2 & 0.2 & 0.2 & 0.8 & 0.2 & 1.42306 & 1.36269 \\
0.5 & 0.2 & 0.2 & 0.2 & 1.2 & 0.2 & 1.52572 & 1.50799 \\
0.5 & 0.2 & 0.2 & 0.2 & 0.5 & 0.0 & 1.42127 & 1.31627 \\
0.5 & 0.2 & 0.2 & 0.2 & 0.8 & 0.5 & 1.42538 & 1.42386 \\
0.5 & 0.2 & 0.2 & 0.2 & 1.2 & 1.0 & 1.42859 & 1.51041 \\
\hline
\end{tabular}

parameter, radiation parameter, Darcy number, magnetic number, and thermal conductivity parameter, while the local Nusselt number increases with the increase of the unsteadiness parameter, the thermal conductivity parameter, and radiation parameter. Also, the local Nusselt number decreases with increase in the viscosity parameter, Darcy number, and the magnetic parameter.

\section{Conclusions}

Numerical solutions have been obtained to study the effects of variable properties and variable heat flux in a laminar flow of an incompressible fluid past an unsteady stretching surface which embedded in a porous medium. The obtained similar ordinary differential equations are solved numerically by using shooting method. All values of parameters corresponding to predominate the shear stress for fluid flow and heat transfer are tabulated for different physical parameters. The obtained highly nonlinear ordinary differential equations which describe our physical problem were solved numerically by using the shooting method. We have found that the local Nusselt number is strongly dependent on the values of the unsteadiness parameter, radiation parameter, and thermal conductivity parameter and dependent to a lesser extent on the values of the magnetic parameter, Darcy number, and viscosity parameter. Also, as the viscosity parameter and the magnetic parameter increase, the rate of heat transfer was found to be decreased. Furthermore, the rate of cooling for the surface was found to be increased as the thermal conductivity parameter, unsteadiness parameter, and radiation parameter increase.

\section{Data Availability}

No data were used to support the findings of this study.

\section{Conflicts of Interest}

The authors declare that they have no conflicts of interest.

\section{Authors' Contributions}

The authors have equal contributions to each part of this paper. All authors read and approved the final manuscript.

\section{Acknowledgments}

Nourhan I. Ghoneim would like to appreciate and thank International Maritime College Oman IMCO, Sultanate of 
Oman, for their efforts through their research support and encouragement.

\section{References}

[1] J. M. Stone, "Astrophysical magnetohydrodynamics," Bulletin of the Astronomical Society of India, vol. 39, pp. 129-143, 2011.

[2] B. Jonathan, MHD: Astrophysical Contexts, Essential Fluid Dynamics for Scientists, Berlin, Germany, 2017.

[3] J. Singh, U. S. Mahabaleshwar, and G. Bognar, "Mass transpiration in nonlinear MHD flow due to porous stretching sheet," Scientific Reports, vol. 9, p. 18484, 2019.

[4] A. Mucoglu and T. S. Chen, "Mixed convection on inclined surfaces," Journal of Heat Transfer, vol. 101, no. 3, pp. 422-426, 1979.

[5] L. J. Grubka and K. M. Bobba, "Heat transfer characteristics of a continuous, stretching surface with variable temperature," Journal of Heat Transfer, vol. 107, no. 1, pp. 248-250, 1985.

[6] M. V. Karwe and Y. Jaluria, "Fluid flow and mixed convection transport from a moving plate in rolling and extrusion processes," Journal of Heat Transfer, vol. 110, no. 3, pp. 655-661, 1988.

[7] C.-H. Chen, "Laminar mixed convection adjacent to vertical, continuously stretching sheets," Heat Mass Transfer, vol. 33, pp. 471-476, 1988.

[8] C. o.-K. Chen and M.-I. Char, "Heat transfer of a continuous, stretching surface with suction or blowing," Journal of Mathematical Analysis and Applications, vol. 135, no. 2, pp. 568-580, 1988.

[9] M. E. Ali, "Heat transfer characteristics of a continuous stretching surface," Wärme - und Stoffübertragung, vol. 29, pp. 227-234, 1994.

[10] S. Bhattacharyya, A. Pal, A. Pal, and A. S. Gupta, "Heat transfer in the flow of a viscoelastic fluid over a stretching surface," Heat and Mass Transfer, vol. 34, no. 1, pp. 41-45, 1998.

[11] M.-I. Char, "Heat transfer in a hydromagnetic flow over a stretching sheet," Wärme- und Stoffübertragung, vol. 29, no. 8, pp. 495-500, 1994.

[12] I. Pop and T.-Y. Na, "Unsteady flow past a stretching sheet," Mechanics Research Communications, vol. 23, no. 4, pp. 413-422, 1996.

[13] E. M. A. Elbashbeshy and M. A. A. Bazid, "Heat transfer over an unsteady stretching surface," Heat and Mass Transfer, vol. 41, no. 1, pp. 1-4, 2004.

[14] I.-C. Liu and H. I. Andersson, "Heat transfer in a liquid film on an unsteady stretching sheet," International Journal of Thermal Sciences, vol. 47, no. 6, pp. 766-772, 2008.

[15] M. A. El-Aziz, "Radiation effect on the flow and heat transfer over an unsteady stretching sheet," International Communications Heat and Mass Transfer, vol. 36, pp. 521-524, 2009.

[16] A. Ishak, R. Nazar, and I. Pop, "Heat transfer over an unsteady stretching permeable surface with prescribed wall temperature," Nonlinear Analysis: Real World Applications, vol. 10, no. 5, pp. 2909-2913, 2009.

[17] M. A. El-Aziz, "Flow and heat transfer over an unsteady stretching surface with Hall effect," Meccanica, vol. 45, pp. 97-109, 2010.

[18] B. K. Dutta, P. Roy, and A. S. Gupta, "Temperature field in flow over a stretching sheet with uniform heat flux," International Communications in Heat and Mass Transfer, vol. 12, no. 1, pp. 89-94, 1985.

[19] C.-L. Chang and Z.-Y. Lee, "Free convection on a vertical plate with uniform and constant heat flux in a thermally stratified micropolar fluid," Mechanics Research Communications, vol. 35, no. 6, pp. 421-427, 2008.

[20] C.-Y. Cheng, "Soret and Dufour effects on natural convection boundary layer flow over a vertical cone in a porous medium with constant wall heat and mass fluxes," International Communications in Heat and Mass Transfer, vol. 38, no. 1, pp. 44-48, 2011.

[21] I.-C. Liu, A. M. Megahed, and H.-H. Wang, "Heat transfer in a liquid film due to an unsteady stretching surface with variable heat flux," ASME Journal of Applied Mechanics, vol. 80, p. 41003, 2013.

[22] S. Rashidi, J. A. Esfahani, and M. Maskaniyan, "Applications of magnetohydrodynamics in biological systems-a review on the numerical studies," Journal of Magnetism and Magnetic Materials, vol. 439, pp. 358-372, 2017.

[23] M. A. A. Mahmoud and A. M. Megahed, "MHD flow and heat transfer in a non-Newtonian liquid film over an unsteady stretching sheet with variable fluid properties," Canadian Journal of Physics, vol. 87, no. 10, pp. 1065-1071, 2009.

[24] K. V. Prasad, K. Vajravelu, P. S. Datti, and B. T. Raju, "MHD flow and heat transfer in a power-law liquid film at a porous surface in the presence of thermal radiation," Journal of Applied Fluid Mechanics, vol. 6, pp. 385-395, 2013.

[25] B. S. Dandapat, B. Santra, and K. Vajravelu, "The effects of variable fluid properties and thermocapillarity on the flow of a thin film on an unsteady stretching sheet," International Journal of Heat and Mass Transfer, vol. 50, no. 5-6, pp. 991996, 2007.

[26] L. J. Crane, "Flow past a stretching plate," Zeitschrift für angewandte Mathematik und Physik ZAMP, vol. 21, no. 4, pp. 645-647, 1970.

[27] J. Singh and R. Bajaj, "Parametric modulation in the TaylorCouette ferrofluid flow," Fluid Dynamics Research, vol. 40, no. 10, pp. 737-752, 2008.

[28] J. Singh and R. Bajaj, "Dean instability in ferrofluids," Meccanica, vol. 51, no. 4, pp. 835-847, 2016.

[29] J. Singh, "A nonlinear shooting method and its application to nonlinear Rayleigh-Bénard convection," ISRN Mathematical Physics, vol. 2013, Article ID 650208, 2013.

[30] K. V. Prasad, D. Pal, and P. S. Datti, "MHD power-law fluid flow and heat transfer over a non-isothermal stretching sheet," Communications in Nonlinear Science and Numerical Simulation, vol. 14, no. 5, pp. 2178-2189, 2009. 